\title{
The Kakimizu complex is simply connected
}

\author{
Jennifer Schultens
}

July 22,2010

\begin{abstract}
In 1992, Osamu Kakimizu defined a complex that has become known as the Kakimizu complex of a knot. Vertices correspond to isotopy classes of minimal genus Seifert surfaces of the knot. Higher dimensional simplices correspond to collections of such classes of Seifert surfaces that admit disjoint representatives. We show that this complex is simply connected.
\end{abstract}

One of the fundamental objects considered in the topological study of knots is the Seifert surface. Interestingly, a knot can have many, and in some cases infinitely many, non-isotopic Seifert surfaces of minimal genus. For instance, a Seifert surface of a connect sum of knots, $K=K_{1} \# K_{2}$, can be spun around a swallow-follow torus of $K$ to yield infinitely many Seifert surfaces that are typically non-isotopic. This phenomenon of non-uniqueness for Seifert surfaces was described early on in [2] and [6]. The Kakimizu complex aims to capture structural information of the set of isotopy classes of Seifert surfaces of a given knot. It is one of several complexes defined by considering isotopy classes of certain submanifolds and disjointness properties of representatives of such isotopy classes.

In 1992, Osamu Kakimizu defined a complex that has become known as the Kakimizu complex of a knot. See [11]. The results in [2] and [6] establish that the Kakimizu complex is nontrivial. A result of M. Scharlemann and A. Thompson, see [19], establishes that the Kakimizu complex is connected. It is conjectured that the Kakimizu complex is contractible.

Distance in the Kakimizu complex can be defined in terms of the number of edges in an edge path between the two vertices, but Kakimizu showed that this is equivalent to a more sophisticated formulation in terms of the universal abelian cover of the knot. Whereas the first definition is the standard definition for distance in a complex, the second provides a more effective means of computing the distance in the Kakimizu complex.

Recent years have seen progress in understanding key facts about the Kakimizu complex: W. Jaco and E. Sedgwick showed that the Kakimizu complex of the knot $K$ is finite if $K$ is atoroidal and has genus at least 2. See [14]. Moreover, R. Wilson showed that, in fact, it suffices to assume that $K$ is atoroidal. See [23]. Results pertaining to specific classes of knots can be found in [9], [15], [17], [18] and [22]. In 
[18], M. Sakuma and K. Shackleton establish concrete diameter bounds and provide an overview of the current understanding of the Kakimizu complex. In particular, they prove that the Kakimizu complex is simply connected for knots of genus 1. A more general understanding of the shape of the Kakimizu complex is highly desirable. Many questions remain unanswered. Though we establish simple connectivity here, see Theorem 6, the conjectured contractibility has yet to be proved.

In Section 1 we provide the formal definition of the Kakimizu complex of a knot and the notion of distance in the Kakimizu complex. Section 2 introduces the concept of a relative least area surface and states two required results. (These results are proved in the appendix.) The heart of the paper lies in Section 3, where we prove two key lemmas that yield information about weighted paths in the Kakimizu complex. Section 4 contains the observation that the Kakimizu complex is a flag complex. In Section 5 we prove the main theorem, Theorem 6, stating that the Kakimizu complex is simply connected. In Section 6 we prove that the Kakimizu complex is contractible in the special case when it is 2-dimensional. We finish with a few remarks in Section 7. The Appendix, by Misha Kapovich, contains the proofs of the theorems about relative least area surfaces required in this context and is of independent interest.

I wish to thank Jesse Johnson, Makoto Sakuma and Ken Shackleton for pointing out a mistake in an earlier argument pertaining to Theorem 6. I also wish to thank Misha Kapovich for helpful conversations and for providing the appendix to this paper. This work was supported, in part, by a grant from the NSF. It was begun at the Max Planck Institute for Mathematics in the Sciences located in Leipzig, Germany and completed at the Max Planck Institute for Mathematics located in Bonn, Germany. I wish to thank the institutes for their hospitality.

\section{Preliminaries}

For basic definitions concerning knots, see [1], [12] and [16]. For basic definitions concerning complexes, see [4]. For a knot $K$ in $\mathbb{S}^{3}$ we will denote an open regular neighborhood of $K$ by $\eta(K)$ and the exterior of $K, \mathbb{S}^{3}-\eta(K)$, by $E(K)$. Recall that a Seifert surface for $K$ is a connected surface with connected boundary representing a generator of $H_{2}(E(K), \partial E(K))$.

Definition 1. The minimal genus Seifert surfaces of a knot $K$ representing a fixed generator of $H_{2}(E(K), \partial E(K))$ form a simplicial complex as follows: 1) Vertices correspond to isotopy classes of minimal genus Seifert surfaces; 2) Edges correspond to pairs of vertices admitting disjoint representatives and, more generally, $n$-dimensional simplices correspond to $(n+1)$-tuples $\left(v_{1}, \ldots, v_{n+1}\right)$ of vertices admitting representatives $\left(S_{1}, \ldots, S_{n+1}\right)$ such that $S_{i} \cap S_{j}=\emptyset$ for all $i<j$.

This complex is called the Kakimizu complex of $K$.

In our discussion here, paths and loops in a simplicial complex will traverse only vertices and edges (not higher dimensional faces). 
Definition 2. The distance between two vertices $v, v^{*}$ in the Kakimizu complex of a $k n o t K$, denoted by $d_{K}\left(v, v^{*}\right)$, is the minimal number of edges in a path connecting the two vertices. The length of a loop is the number of edges in the loop.

One of the fundamental results concerning the Kakimizu complex is due to M. Scharlemann and A. Thompson. See [19]. It refers to the intersection number of surfaces. Recall that the intersection number of a pair of surfaces $\left(S, S^{*}\right), i\left(S, S^{*}\right)$, is defined to be the least number of components of intersection of pairs of surfaces isotopic to $\left(S, S^{*}\right)$ that have transverse intersection. In the language here, the theorem can be formulated as follows:

Theorem 1. (Scharlemann-Thompson) The Kakimizu complex of a knot $K$ in $\mathbb{S}^{3}$ is connected. Moreover, given two Seifert surfaces $S, S^{*}$, the distance of the corresponding vertices in the Kakimizu complex is bounded above by $i\left(S, S^{*}\right)+1$.

\section{A few facts about least area surfaces}

Our arguments will rely extensively on the use of (analytic) least area surfaces. In addition, we will be interested in the behavior of our surfaces near the boundary of our knot complements.

Definition 3. Let $M$ be a compact irreducible smooth manifold with boundary. A relative Riemannian metric on $M$ is a Riemannian metric such that $\partial M$ is strictly convex. Suppose that $\partial M$ consists of tori and let $\mathcal{J}$ be a smooth foliation of $\partial M$ by closed curves. A properly embedded surface $F$ in $M$ is relative least area if the following hold: 1) $\partial F \subset \mathcal{J}$; 2) The surface $F$ minimizes area over all surfaces in its proper isotopy class subject to the constraint $\partial F \subset \mathcal{J}$.

Let $M$ be a compact irreducible smooth manifold with $\partial M$ consisting of tori and with a relative Riemannian metric. In what follows we will always assume that a foliation of $\partial M$ is fixed. In the case of a knot complement, we will assume that $\mathcal{J}$ consists of preferred longitudes. When we consider relative least area surfaces, they will be considered with respect to this fixed foliation. Let $F$ be a properly embedded surface in $M$. We denote the proper isotopy class of $F$ by $[F]$. Furthermore, we denote the area of $F$ by $A(F)$ and the area of a relative least area representative of $[F]$ by $A([F])$.

In what follows we will specify a path by the vertices it traverses, e.g., $v^{1}, \ldots, v^{n}$.

Definition 4. The complexity of a path $v^{1}, \ldots, v^{n}$ in the Kakimizu complex is the ordered pair $(n, a)$ where

$$
a=A\left(\left[S^{1}\right]\right)+\cdots+A\left(\left[S^{n}\right]\right)
$$

and $S^{1}, \ldots, S^{n}$ are representatives of $v^{1}, \ldots, v^{n}$ respectively. We give the set of complexities the lexicographic order.

In the special case where $n=1$, we denote the complexity of the vertex $v^{1}$ by $c\left(v^{1}\right)$. 
The following theorems are proved in the appendix.

Theorem 2. Let E be a compact irreducible 3-manifold endowed with a relative Riemannian metric and let $F$ be a properly embedded compact incompressible surface in $E$. Then there exists a relative least area representative in the proper isotopy class of $F$.

This is Corollary 11 from the appendix.

Theorem 3. If there is a homotopically nontrivial loop of length $n>0$ in the Kakimizu complex of a knot $K$, then there is a homotopically nontrivial loop of smallest complexity of length $n$.

This is Corollary 13 in the appendix.

Theorem 4. Let E be a compact irreducible 3-manifold endowed with a relative Riemannian metric and let $F_{1}, F_{2}$ be properly embedded relative least area incompressible surfaces in $E$ with disjoint representatives in their proper isotopy classes. Then either $F_{1}, F_{2}$ are disjoint or they coincide.

This is Theorem 12 in the appendix. (It is a variant of [7, Theorem 6.2].)

Two of the standard tools used in conjunction with least area surfaces are "exchangeroundoff" and the Meeks-Yau trick. The term "exchange-roundoff" refers to the fact that cut-and-paste along a pair of transverse least area surfaces yields a pair of lower area least area surfaces. (The area remains the same after cut-and-paste, but decreases after roundoff.) See Figure 1.

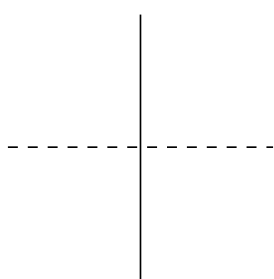

1

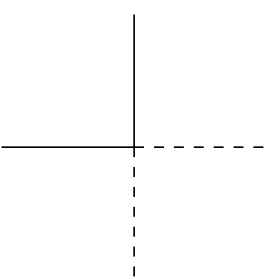

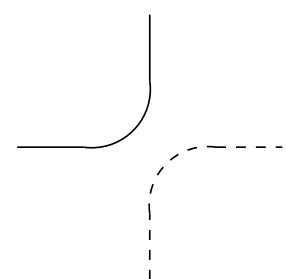

3

Figure 1: (1) Cross-section of surfaces before exchange. (2) Cross-section of surfaces after exchange. (3) Cross-section of surfaces after roundoff.

A pair of least area surfaces need not be transverse. For instance, there can be saddle intersections. For specific examples, see [7]. Additionally, a pair of relative least area surfaces can share components of their boundary. The Meeks-Yau trick allows us to skirt this issue. For an illustration, see the discussion in Case 2 of the proof of Lemma 2 below. 


\section{Key Lemmas}

The following lemmas are crucial in the proof of the main theorem (Theorem 6). They allow us to refine the construction of Scharlemann and Thompson [19]. We denote the symmetric difference of two sets, $X, Y$, by $X \Delta Y$. Recall that $X \Delta Y=$ $(X \cup Y) \backslash(X \cap Y)$.

Lemma 1. Let $K$ be a knot in $\mathbb{S}^{3}$ and suppose that $S, S^{+1}, S^{-1}$ are minimal genus Seifert surfaces for $K$ such that the following hold:

1. $S$ is disjoint from $S^{+1} \cup S^{-1}$;

2. $S^{-1} \cap S^{+1} \neq \emptyset$;

3. The intersection between $S^{-1}$ and $S^{+1}$ is transverse and $\partial S^{-1} \cap \partial S^{+1}=\emptyset$;

4. There are no disk components in $S^{-1} \Delta S^{+1}$.

Then there are two minimal genus Seifert surfaces $S^{u p}, S^{\text {down }}$ for $K$ such that (a)

$$
\begin{gathered}
S \cap S^{\text {up }}=\emptyset, S \cap S^{\text {down }}=\emptyset,\left(S^{+1} \cup S^{-1}\right) \cap S^{u p}=\emptyset, \\
\left(S^{+1} \cup S^{-1}\right) \cap S^{\text {down }}=\emptyset, S^{\text {up }} \cap S^{\text {down }}=\emptyset .
\end{gathered}
$$

Moreover, (b) if there is a surface $F$ disjoint from $S^{+1} \cup S^{-1}$, then it is also disjoint from $S^{\text {down }}, S^{\text {up }}$.

Suppose furthermore that $E(K)$ is endowed with a relative Riemannian metric and that $S^{+1}, S^{-1}$ are relative least area surfaces. Then (c)

$$
A\left(S^{-1}\right)+A\left(S^{+1}\right)>A\left(\left[S^{\text {down }}\right]\right)+A\left(\left[S^{u p}\right]\right) .
$$

Proof: We will construct $S^{\text {up }}, S^{\text {down }}$ explicitly by using the universal abelian cover $M(K)$ of $E(K)$. Let $\tau$ be a generator of the group of covering translations and let $S_{0}$ be a lift of $S$ to $M(K)$. Set

$$
S_{1}=\tau\left(S_{0}\right)
$$

Denote the component of

$$
M(K) \backslash\left(S_{0} \cup S_{1}\right)
$$

that lies between $S_{0}$ and $S_{1}$ by $C$ and note that $C$ is homeomorphic to $E(K) \backslash S$ via the restriction of the covering map $M(K) \rightarrow E(K)$. See Figure 2. In particular, there are lifts $S_{0}^{+1}, S_{0}^{-1}$ of $S^{+1}, S^{-1}$, respectively, in $C$.

Denote the two components of $M(K) \backslash S_{0}^{-1}$ by $M_{-}^{-1}$ and $M_{+}^{-1}$, with $M_{+}^{-1}$ the component above $S_{0}^{-1}$, i.e., the component containing $S_{1}$. Similarly, denote the two components of $M(K) \backslash S_{0}^{+1}$ by $M_{-}^{+1}$ and $M_{+}^{+1}$, with $M_{+}^{+1}$ the component above $S_{0}^{+1}$, i.e., the component containing $S_{1}$. Recall that the frontier of a subset $H$, denoted by $\operatorname{fr}(H)$, is the closure of $H$ (in the ambient space) minus the interior of $H$. 


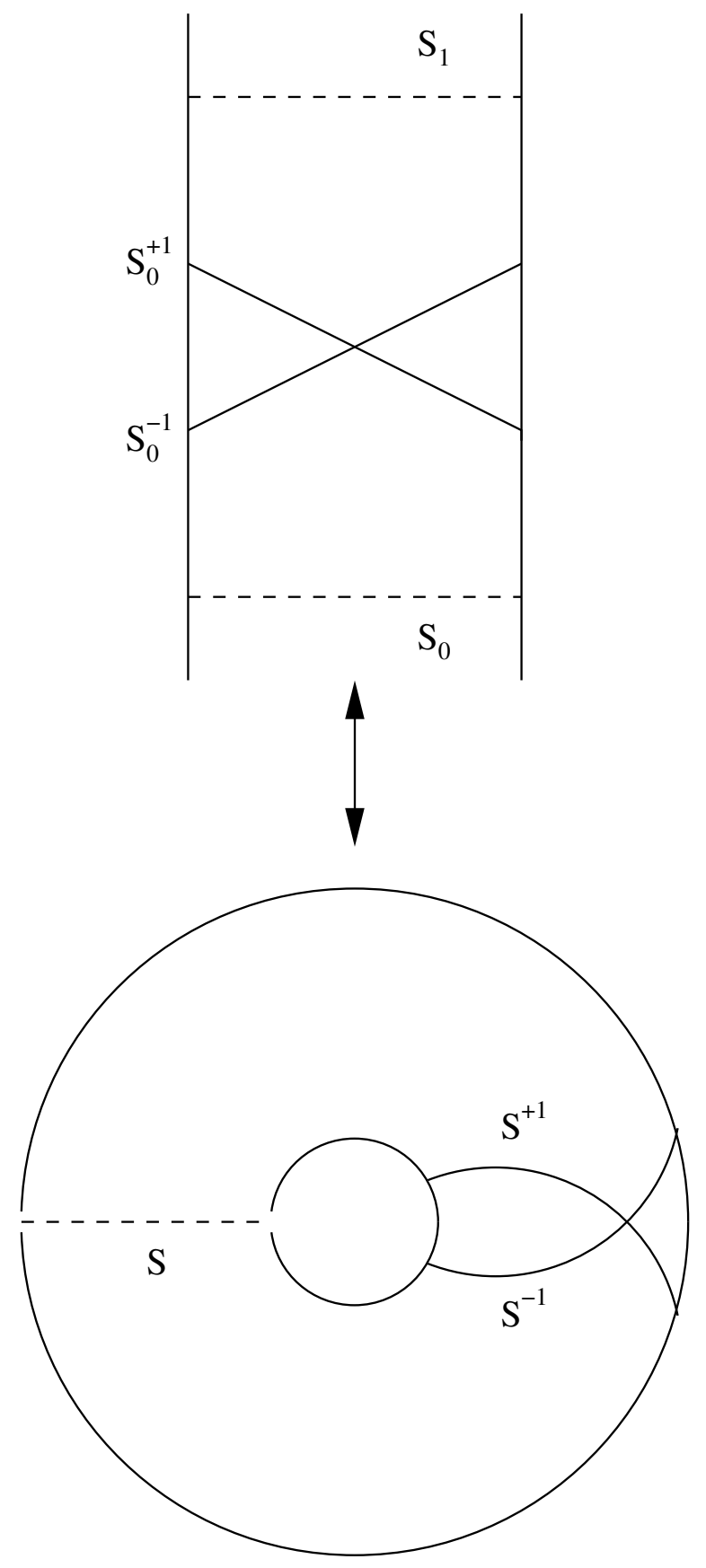

Figure 2: $M(K)$ and $E(K)$ 
Set

$$
\tilde{B}^{\text {down }}=f r\left(M_{-}^{-1} \cap M_{-}^{+1}\right)
$$

and

$$
\tilde{B}^{u p}=f r\left(M_{+}^{-1} \cap M_{+}^{+1}\right) .
$$

Further, let $\tilde{T}^{\text {down }}$ be a small pushoff of $\tilde{B}^{\text {down }}$ into $M_{-}^{-1} \cap M_{-}^{+1}$ and $\tilde{T}^{u p}$ a small pushoff of $\tilde{B}^{u p}$ into $M_{+}^{-1} \cap M_{+}^{+1}$. See Figure 3 . Let $B^{\text {up }}, B^{\text {down }}, T^{\text {up }}, T^{\text {down }}$ denote the (homeomorphic) projections of $\tilde{B}^{u p}, \tilde{B}^{\text {down }}, \tilde{T}^{\text {up }}, \tilde{T}^{\text {down }}$ to the manifold $E(K)$. (Note that these surfaces could be disconnected.)

Claim 1: $T^{u p}, T^{\text {down }}$ each contain one Seifert surface.

Let $\gamma$ be an oriented simple closed curve on $\partial E(K)$ that generates the homology of $E(K)$ and let $\tilde{\gamma}$ be its lift to $M(K)$. The algebraic intersection number of $\gamma$ with $\partial S, \partial S^{+1}, \partial S^{-1}$, respectively, is 1 . Hence the algebraic intersection number of $\tilde{\gamma}$ with $\partial S_{0}^{+1}, \partial S_{0}^{-1}$, respectively, is also 1, because of the homeomorphism between $C$ and $E(K) \backslash S$. Note that one component of $\partial S_{0}^{-1} \cup \partial S_{0}^{+1}$ lies in $\tilde{B}^{u p}$ and the other lies in $\tilde{B}^{\text {down}}$. It follows that the algebraic intersection number of $\tilde{\gamma}$ with $\partial \tilde{T}^{u p}, \partial \tilde{T}^{\text {down }}$, respectively, is 1 . This in turn means that the algebraic intersection number of $\gamma$ with $T^{u p}, T^{\text {down }}$, respectively, is 1 , because of the homeomorphism between $C$ and $E(K) \backslash S$. It follows that $T^{u p}, T^{\text {down }}$ each contain one Seifert surface. This proves Claim 1.

Denote the Seifert surfaces in $T^{\text {down }}, T^{u p}$ by $S^{\text {down }}, S^{u p}$, respectively. Then

$$
\begin{gathered}
S \cap S^{\text {down }}=\emptyset \\
S \cap S^{\text {up }}=\emptyset \\
\left(S^{+1} \cup S^{-1}\right) \cap S^{\text {down }}=\emptyset \\
\left(S^{+1} \cup S^{-1}\right) \cap S^{\text {up }}=\emptyset \\
S^{\text {down }} \cap S^{\text {up }}=\emptyset
\end{gathered}
$$

Claim 2: $S^{u p}, S^{\text {down }}$ are minimal genus Seifert surfaces of $K$.

Note that

$$
\chi\left(T^{u p}\right)+\chi\left(T^{\text {down }}\right)=\chi\left(\tilde{T}^{u p}\right)+\chi\left(\tilde{T}^{\text {down }}\right)
$$

since $T^{u p} \cup T^{\text {down }}$ is homeomorphic to $\tilde{T}^{u p} \cup \tilde{T}^{\text {down }}$. Also,

$$
\chi\left(\tilde{T}^{u p}\right)+\chi\left(\tilde{T}^{\text {down }}\right)=\chi\left(S_{0}^{+1}\right)+\chi\left(S_{0}^{-1}\right),
$$

since $\tilde{T}^{u p}$ (resp. $\tilde{T}^{\text {down }}$ ) is isotopic to $\tilde{B}^{u p}\left(\right.$ resp. $\left.\tilde{B}^{\text {down }}\right)$, and

$$
\tilde{B}^{u p} \cup \tilde{B}^{\text {down }}=S_{0}^{+1} \cup S_{0}^{-1} .
$$

Finally,

$$
\chi\left(S_{0}^{+1}\right)+\chi\left(S_{0}^{-1}\right)=\chi\left(S^{-1}\right)+\chi\left(S^{+1}\right)
$$




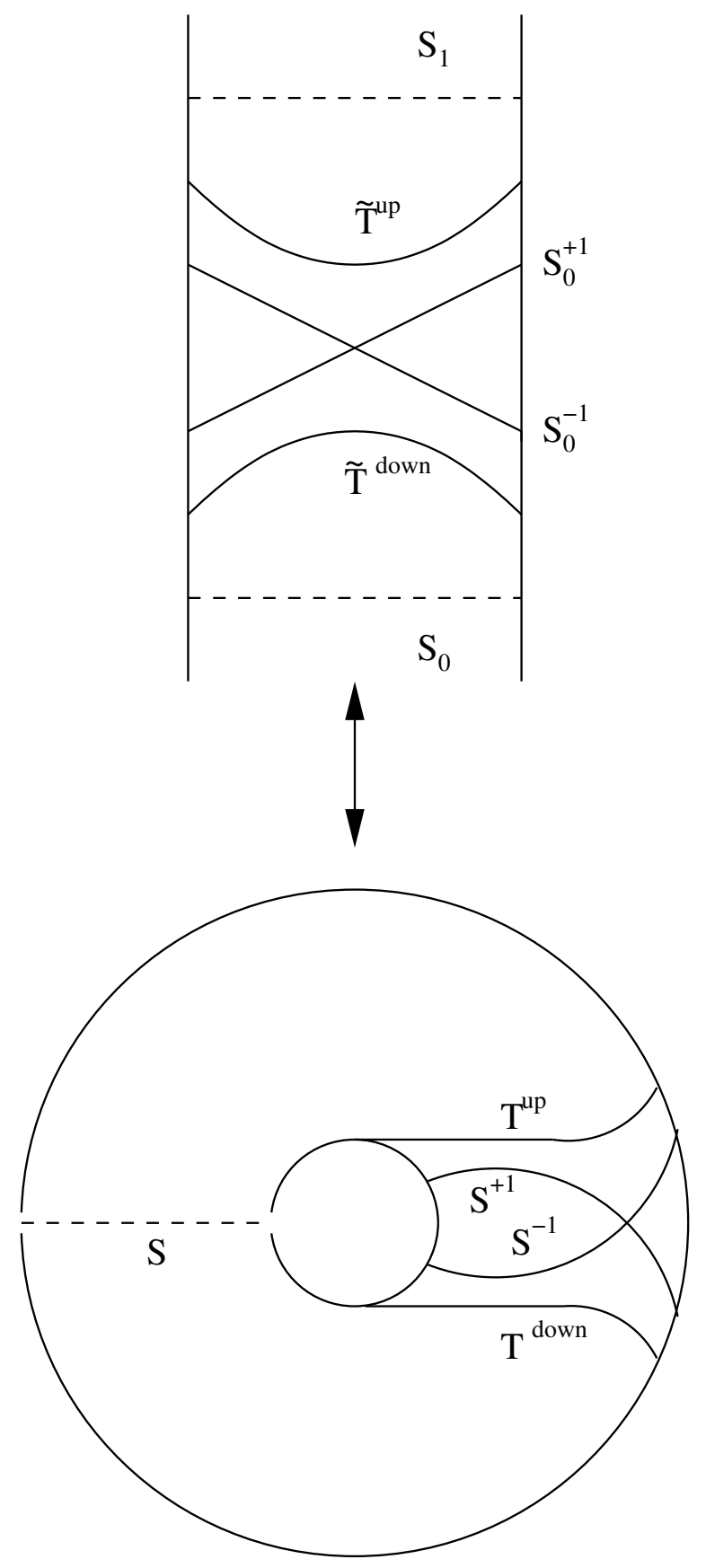

Figure $3: \tilde{T}^{\text {down }}$ and $\tilde{T}^{u p}$ 
since $S_{0}^{-1} \cup S_{0}^{+1}$ is homeomorphic to $S^{-1} \cup S^{+1}$. Therefore

$$
\chi\left(T^{\text {up }}\right)+\chi\left(T^{\text {down }}\right)=\chi\left(S^{-1}\right)+\chi\left(S^{+1}\right) .
$$

There are no disks in $S^{-1} \Delta S^{+1}$, hence there are no disks in $S_{0}^{-1} \Delta S_{0}^{+1}$. Thus there are no disks or 2 -spheres in $\tilde{T}^{u p}, \tilde{T}^{\text {down }}$. Therefore there are no disks or 2 -spheres in $T^{u p}, T^{\text {down }}$. So

$$
\chi\left(S^{\text {up }}\right)+\chi\left(S^{\text {down }}\right) \geq \chi\left(T^{\text {up }}\right)+\chi\left(T^{\text {down }}\right)
$$

since

$$
S^{\text {up }} \cup S^{\text {down }} \subset T^{\text {up }} \cup T^{\text {down }} \text {. }
$$

Set $g=\operatorname{genus}(K)$ (the minimal genus of a Seifert surface of $K$ ). Then

$$
\chi\left(S^{-1}\right)+\chi\left(S^{+1}\right)=1-2 g+1-2 g=2-4 g,
$$

since $S^{+1}$ and $S^{-1}$ are minimal genus Seifert surfaces. Note also that

$$
\chi\left(S^{\text {down }}\right) \leq 1-2 g, \chi\left(S^{\text {up }}\right) \leq 1-2 g .
$$

By this observation and the above computation,

$$
2-4 g \geq \chi\left(S^{\text {up }}\right)+\chi\left(S^{\text {down }}\right) \geq 2-4 g .
$$

It follows that

$$
\chi\left(S^{\text {up }}\right)=\chi\left(S^{\text {down }}\right)=1-2 g .
$$

This proves Claim 2.

This proves part (a) of the Lemma. Part (b) follows from the construction. We now prove part (c) of the Lemma.

Suppose that $E(K)$ is endowed with a relative Riemannian metric. Equip $M(K)$ with the pull-back of this metric. Since $C$ is isometric to $E(K) \backslash S$ via the restriction of the covering map $M(K) \rightarrow E(K)$, we have

$$
A\left(S^{-1}\right)+A\left(S^{+1}\right)=A\left(S_{0}^{-1}\right)+A\left(S_{0}^{+1}\right)
$$

and

$$
A\left(\tilde{B}^{\text {down }}\right)+A\left(\tilde{B}^{\text {up }}\right)=A\left(B^{\text {down }}\right)+A\left(B^{\text {up }}\right) .
$$

By the construction of $\tilde{B}^{\text {down }}, \tilde{B}^{u p}$,

$$
A\left(S_{0}^{-1}\right)+A\left(S^{+1}\right)=A\left(\tilde{B}^{\text {down }}\right)+A\left(\tilde{B}^{u p}\right) .
$$

Note that the surfaces $B^{\text {down }}, B^{u p}$ are not smooth, while the relative least area surfaces in their respective isotopy classes are necessarily smooth. Therefore,

$$
A\left(B^{\text {down }}\right)>A\left(\left[S^{\text {down }}\right]\right), \quad A\left(B^{\text {up }}\right)>A\left(\left[S^{\text {up }}\right]\right) .
$$

It follows that

$$
A\left(S^{-1}\right)+A\left(S^{+1}\right)=A\left(\tilde{B}^{\text {down }}\right)+A\left(\tilde{B}^{u p}\right)>A\left(\left[S^{\text {down }}\right]\right)+A\left(\left[S^{\text {up }}\right]\right) .
$$

Lemma 2 below reinterprets Lemma 1 above in the context of the Kakimizu complex. 
Lemma 2. Let $K$ be a knot in $\mathbb{S}^{3}$ and suppose that $v, v^{+1}, v^{-1}$ are vertices in the Kakimizu complex of $K$ such that

1.

$$
d_{K}\left(v, v^{+1}\right)=1, d_{K}\left(v, v^{-1}\right)=1
$$

2.

$$
d_{K}\left(v^{-1}, v^{+1}\right)=2
$$

3. The complexity of $v$ is no smaller than the complexity of $v^{+1}, v^{-1}$, respectively.

Then there are vertices $v^{\text {down }}, v^{u p}$ in the Kakimizu complex of $K$ such that (a)

$$
\begin{gathered}
d_{K}\left(v, v^{\text {down }}\right) \leq 1, d_{K}\left(v, v^{u p}\right) \leq 1, d_{K}\left(v^{+1}, v^{u p}\right) \leq 1, d_{K}\left(v^{-1}, v^{u p}\right) \leq 1, \\
d_{K}\left(v^{+1}, v^{\text {down }}\right) \leq 1, d_{K}\left(v^{-1}, v^{\text {down }}\right) \leq 1, d_{K}\left(v^{\text {down }}, v^{\text {up }}\right) \leq 1
\end{gathered}
$$

Moreover, (b) if there is a vertex $w$ such that

$$
d_{K}\left(w, v^{+1}\right)=1, d_{K}\left(w, v^{-1}\right)=1
$$

then

$$
d_{K}\left(w, v^{\text {down }}\right) \leq 1, d_{K}\left(w, v^{u p}\right) \leq 1
$$

Furthermore, (c) the complexity of either $v^{u p}$ or of $v^{\text {down }}$ is strictly less than that of $v$.

Proof: Endow $E(K)$ with a relative Riemannian metric and let $\mathcal{J}$ be a smooth foliation of $\partial E(K)$ by preferred longitudes. Let $S, S^{+1}, S^{-1}$ be relative least area representatives of $v, v^{+1}, v^{-1}$ which exist by Theorem 2 . We will say that $S^{+1}, S^{-1}$ are in general position if they intersect transversely and their boundaries in $\partial E(K)$ are disjoint.

Case 1: $S^{-1}, S^{+1}$ are in general position.

Since

$$
\begin{gathered}
d_{K}\left(v, v^{+1}\right)=1, d_{K}\left(v, v^{-1}\right)=1 \\
d_{K}\left(v^{-1}, v^{+1}\right)=2
\end{gathered}
$$

we have

$$
S^{-1} \cap S^{+1} \neq \emptyset
$$

and Theorem 4 gives us

$$
S \cap S^{+1}=\emptyset, S \cap S^{-1}=\emptyset .
$$

By [7, Lemma 1.2] there are no disks in $S^{-1} \Delta S^{+1}$. (Such a disk would yield a "product region" in the sense of [7].) Thus all hypotheses of Lemma 1 are satisfied. Let $v^{u p}, v^{\text {down }}$ be the vertices in the Kakimizu of $K$ corresponding to $S^{u p}, S^{\text {down }}$. Then 
parts (a) and (b), respectively, follow from parts (a) and (b), respectively, of Lemma 1.

Furthermore, the statement about complexities follows because say,

$$
A\left(\left[S^{\text {down }}\right]\right) \geq A\left(\left[S^{\text {up }}\right]\right)
$$

and thus

$$
\begin{gathered}
A(S) \geq \max \left\{A\left(S^{-1}\right), A\left(S^{+1}\right)\right\} \geq \frac{1}{2}\left(A\left(S^{-1}\right)+A\left(S^{+1}\right)\right)> \\
\frac{1}{2}\left(A\left(\left[S^{\text {up }}\right]\right)+A\left(\left[S^{\text {down }}\right]\right)\right) \geq A\left(\left[S^{\text {up }}\right]\right) .
\end{gathered}
$$

Hence

$$
A(S)>A\left(\left[S^{u p}\right]\right)
$$

This proves part (c).

Case 2: $S^{-1}, S^{+1}$ are not in general position.

In this case we apply the Meeks-Yau trick as described in [7, Proof of Lemma 1.3]: Let $x \in S^{-1} \cap S^{+1}$ be an interior point of $E(K)$ where the surfaces intersect transversely. Let $D_{-1} \subset S^{-1}, D_{+1} \subset S^{+1}$ be small disks about $x$, both contained in the interior of $E(K)$, so that the intersection $\alpha=D_{1} \cap D_{-1}$ is a (smooth) arc of transverse intersection between these disks. Then there are portions of the surfaces $B^{u p}, B^{\text {down }}$ near $x$ that are obtained by cutting $D_{+1}$ and $D_{-1}$ along $\alpha$ and pasting them together. The results are two piecewise-smooth disks $D^{u p} \subset B^{u p}$ and $D^{\text {down }} \subset B^{\text {down }}$. Neither disk is smooth along $\alpha$. Therefore, by "rounding off" these disks along $\alpha$ and keeping their boundaries fixed, we obtain two disks whose total area is less than

$$
\operatorname{Area}\left(D_{1}\right)+\operatorname{Area}\left(D_{2}\right)-\epsilon, \quad \epsilon>0 .
$$

Next, take a surface $S^{-1}(t)$ which is sufficiently close to $S^{-1}$ in the $C^{1}$-topology, so that:

1. $\partial S^{-1}(t) \subset \partial E(K)$ lies in $\mathcal{J}$ and is disjoint from $\partial S^{+1}$.

2. $S^{-1}(t)$ intersects $S^{+1}$ transversely.

3. $D_{-1} \subset S^{-1}(t)$.

4. $\operatorname{Area}\left(S^{-1}(t)\right)<\operatorname{Area}\left(S^{-1}\right)+\epsilon$.

Now apply the argument from Case 1 to the surfaces $S^{-1}(t)$ and $S^{+1}$. As explained in [7, Proof of Lemma 1.3], there are no "product regions" between the new surfaces. In particular, since $S^{+1}$ and $S^{-1}$ are incompressible and $E(K)$ is irreducible, it follows that the symmetric difference $S^{-1}(t) \Delta S^{+1}$ contains no disks. Construct surfaces $B^{u p}(t)$ and $B^{\text {down }}(t)$ and $S^{\text {up }}(t), S^{\text {down }}(t)$ in the same way as before. Since by "rounding-off" $B^{\text {up }}(t)$ and $B^{\text {down }}(t)$ we loose more total area than we have gained by replacing $S^{-1}$ with $S^{-1}(t)$, we conclude that

$$
A\left(\left[S^{u p}(t)\right]\right)+A\left(\left[S^{\text {down }}(t)\right]\right)<A\left(S^{-1}\right)+A\left(S^{+1}\right) .
$$

The remainder of the argument follows as before. 


\section{The Kakimizu complex is flag}

We will use the following Theorem to prove the main theorem (Theorem 6), but it is interesting in its own right. Recall that a simplicial complex is flag if it contains no empty simplices, i.e., if it contains an $n$-simplex whenever it contains the $(n-1)$ skeleton of the simplex.

Theorem 5. The Kakimizu complex of a knot is flag.

Proof: Let $K$ be a knot. Endow $E(K)$ with a relative Riemannian metric. If the Kakimizu complex of $K$ contains the 1-skeleton of the simplex $\sigma$, then, by definition, there are disjoint minimal genus Seifert surfaces representing any pair of vertices in $\sigma$. Hence, if we choose least area representatives for the vertices, it follows from Theorem 4 that these representatives are simultaneously disjoint. Thus $\sigma$ belongs to the Kakimizu complex.

\section{The Kakimizu complex is simply connected}

We here prove that the Kakimizu complex is simply connected. Recall that paths and loops in the Kakimizu complex traverse only vertices and edges (not higher dimensional simplices). Recall that we specify paths by the vertices they traverse, e.g., $v^{1}, \ldots, v^{n}$. In the case of loops, we abuse notation slightly and write $0,1, \ldots, n+1$ when we really mean $0 \bmod n, \ldots, n+1 \bmod n$.

Theorem 6. Let $K$ be a knot in $\mathbb{S}^{3}$. The Kakimizu complex of $K$ is simply connected.

Proof: Let $v^{1}, \ldots, v^{n}$ be the vertices in a loop in the Kakimizu complex of $K$.

Claim 1: If $d_{K}\left(v^{i-1}, v^{i+1}\right)=1$ for some $i$, then $v^{1}, \ldots, v^{n}$ is homotopic to a shorter loop.

If $d_{K}\left(v^{i-1}, v^{i+1}\right)=1$ and $n=3$, then the loop spans a 2-simplex in the Kakimizu complex by Theorem 5 and is hence homotopic to a single vertex. If $d_{K}\left(v^{i-1}, v^{i+1}\right)=1$ and $n>3$, then $v^{i-1}, v^{i}, v^{i+1}$ still spans a 2-simplex. Hence the loop $v^{1}, \ldots, v^{n}$ is homotopic to the loop obtained by replacing $v^{i-1}, v^{i}, v^{i+1}$ with $v^{i-1}, v^{i+1}$. This proves Claim 1.

We will henceforth assume that

$$
d_{K}\left(v^{i-1}, v^{i+1}\right)=2 \forall i .
$$

Claim 2: If $c\left(v^{i}\right) \geq \max \left\{c\left(v^{i-1}\right), c\left(v^{i+1}\right)\right\}$, then the complexity of the loop $v^{1}, \ldots, v^{n}$ is not minimal among loops homotopic to $v^{1}, \ldots, v^{n}$.

Under the assumption $c\left(v^{i}\right) \geq \max \left\{c\left(v^{i-1}\right), c\left(v^{i+1}\right)\right\}$, Lemma 2 furnishes two vertices $v^{u p}, v^{\text {down }}$ in the Kakimizu complex such that

$$
d_{K}\left(v^{i+1}, v^{u p}\right) \leq 1, d_{K}\left(v^{i-1}, v^{u p}\right), d_{K}\left(v^{i+1}, v^{\text {down }}\right) \leq 1, d_{K}\left(v^{i-1}, v^{\text {down }}\right),
$$




$$
d_{K}\left(v^{i}, v^{\text {down }}\right) \leq 1, d_{K}\left(v^{i}, v^{u p}\right) \leq 1
$$

and such that the complexity of say, $v^{u p}$, is strictly less than that of $v^{i}$.

In particular, the two loops

$$
\begin{aligned}
& v^{i-1}, v^{i}, v^{u p} \\
& v^{i}, v^{i+1}, v^{u p}
\end{aligned}
$$

bound 2-simplices in the Kakimizu complex of $K$, so

$$
v^{1}, \ldots, v^{n}
$$

is homotopic to

$$
v^{1}, \ldots, v^{i-1}, v^{u p}, v^{i+1}, \ldots, v^{n}
$$

See Figure 4.

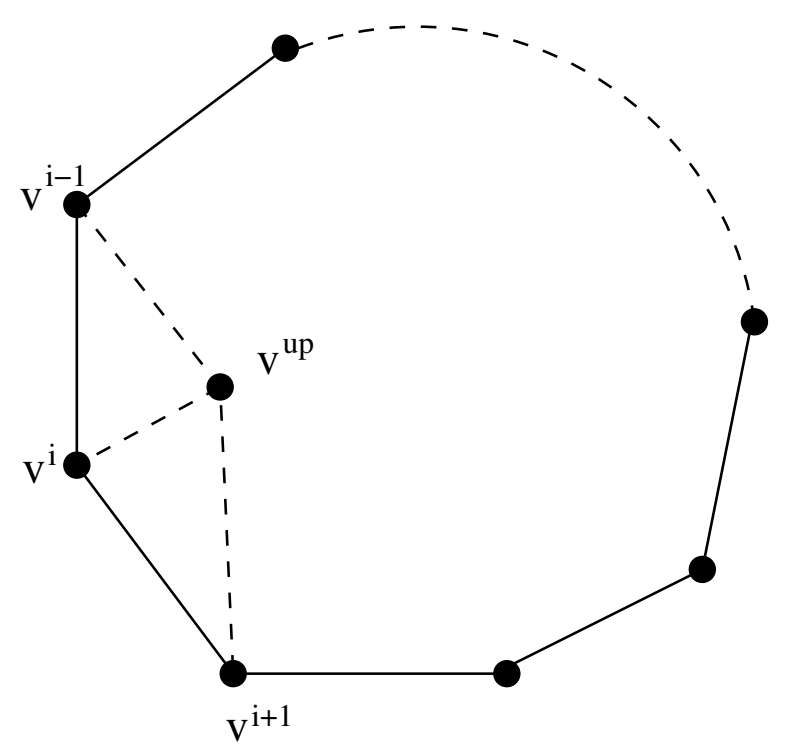

Figure 4: A homotopy

Since $c\left(v^{u p}\right)<c\left(v^{i}\right)$, the complexity of this path is strictly smaller than that of the path $v^{1}, \ldots, v^{n}$. This proves Claim 2 .

Now suppose that there is a homotopically nontrivial loop in the Kakimizu complex of $K$. By Theorem 3, there is a homotopically nontrivial loop $v^{1}, \ldots, v^{n}$ of smallest complexity. Then Claim 2 tells us that

$$
c\left(v^{i}\right)<\max \left\{c\left(v^{i-1}\right), c\left(v^{i+1}\right)\right\} \forall i .
$$

But this is impossible. Thus the Kakimizu complex of $K$ is simply connected. 


\section{A note on contractibility}

We recall some of the standard terminology for simplicial complexes: The link of a vertex $v$ in a simplicial complex $X$, denoted by $X_{v}$, is the union of all simplices disjoint from $v$ that together with $v$ span a simplex in $X$. The star of a vertex $v$ in a simplicial complex $X$ is the union of all simplices in $X$ that contain $v$. A 2-dimensional simplicial complex $X$ is said to be locally $k$-large if for every vertex $v \in X$, every homotopically nontrivial loop in $X_{v}$ has length at least $k$.

The following theorem is classical. It follows from Propositions II.4.1 (CartanHadamard Theorem) and II.5.25 in [4]. See Section 7 for a more general version.

Theorem 7. The universal cover of a 2-dimensional connected locally 6-large simplicial complex is contractible.

Lemma 3. A homotopically nontrivial loop in the link of a vertex in the Kakimizu complex must have length at least 5.

Proof: Loops of length 3 in the Kakimizu complex bound 2-simplices, by Theorem 5 . Hence it suffices to show that there are no homotopically nontrivial loops of length 4 in the link of a vertex. Suppose that $v^{1}, v^{2}, v^{3}, v^{4}$ is a loop in the link, $X_{v}$, of the vertex $v$. We argue as in the proof of Theorem 6 . The same reasoning as in Claim 1 of the proof of Theorem 6 tells us that we need only consider the case in which

$$
d_{K}\left(v^{i-1}, v^{i+1}\right)=2 \forall i \text {. }
$$

The reasoning in Claim 2 of the proof of Theorem 6, tells us that there is a loop homotopic to $v^{1}, \ldots, v^{4}$ of lesser complexity. By part (b) of Lemma 2, the vertex $v^{u p}$ that replaces one of $v^{1}, \ldots, v^{4}$ lies in the star of $v$. It either lies in the link of $v$ or it is equal to $v$. In the latter case we must examine how this comes about. Following the reasoning in Claim 2 of the proof of Theorem 6, we apply Lemma 2 to the vertices $v^{i}, v^{i+1}, v^{i-1}$ in place of the vertices $v, v^{+1}, v^{-1}$ to obtain vertices $v^{\text {down }}, v^{u p}$ such that

$$
\begin{gathered}
d_{K}\left(v^{i+1}, v^{\text {down }}\right) \leq 1, d_{K}\left(v^{i-1}, v^{\text {down }}\right) \leq 1, d_{K}\left(v^{i+1}, v^{u p}\right) \leq 1, d_{K}\left(v^{i-1}, v^{u p}\right) \leq 1, \\
d_{K}\left(v^{i}, v^{\text {down }}\right) \leq 1, d_{K}\left(v^{i}, v^{\text {up }}\right) \leq 1, d_{K}\left(v, v^{\text {down }}\right) \leq 1, d_{K}\left(v, v^{\text {up }}\right) \leq 1
\end{gathered}
$$

and (by part (b) of Lemma 2) also that

$$
d_{K}\left(v^{i+2}, v^{\text {down }}\right) \leq 1, d_{K}\left(v^{i+2}, v^{u p}\right) \leq 1
$$

Two cases need to be considered:

Case 1: $v^{u p}=v$ and $v^{\text {down }} \neq v$.

If $v^{\text {down }}$ is equal to one of the $v^{j}$, then the loop $v^{1}, v^{2}, v^{3}, v^{4}$ breaks into two loops of length 3. The latter bound 2-simplices by Lemma 5. Hence we will assume that 
$v^{\text {down }}$ is not equal to one of the $v^{j}$. Now $v^{i-1}, v^{i}, v^{\text {down }}$ and $v^{i}, v^{\text {down }}, v^{i+1}$ are loops of length 3 and hence bound 2-simplices by Theorem 5 . It follows that our loop

$$
v^{i-1}, v^{i}, v^{i+1}, v^{i+2}
$$

is homotopic to

$$
v^{i-1}, v^{\text {down }}, v^{i+1}, v^{i+2}
$$

in $X_{v}$. In addition, both $v^{\text {down }}, v^{i+1}, v^{i+2}$ and $v^{i+2}, v^{i-1}, v^{\text {down }}$ bound 2-simplices by Lemma 5. Thus $v^{i-1}, v^{\text {down }}, v^{i+1}, v^{i+2}$ and hence $v^{i-1}, v^{i}, v^{i+1}, v^{i+2}$, i.e., $v^{1}, \ldots, v^{4}$, is homotopically trivial in $X_{v}$. See Figure 5 .

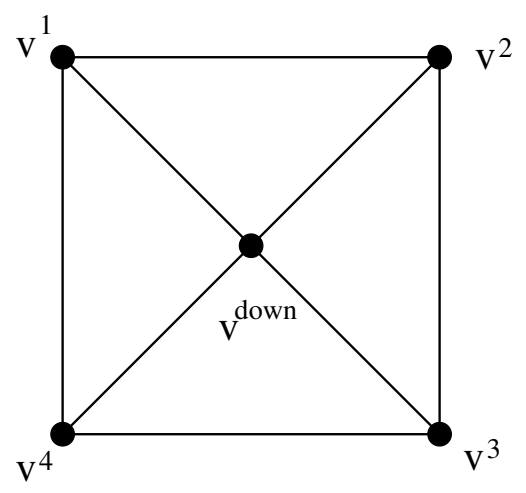

Figure 5: A homotopically trivial loop

Case 2: $v^{u p}=v^{\text {down }}=v$.

In this case we must argue differently. Let $F$ be a representative of $v, F_{0}$ a lift of $F$ to $M(K), F_{1}=\tau\left(F_{0}\right)$ and $C$ the component of

$$
M(K) \backslash\left(F_{0} \cup F_{1}\right)
$$

that lies between $F_{0}$ and $F_{1}$. Recall the notation $S_{0}^{j}, \tilde{T}^{\text {up }}, \tilde{T}^{\text {down }}, S^{\text {up }}, S^{\text {down }}$ from the proof of Lemma 1 and let $\tilde{S}^{\text {up }}, \tilde{S}^{\text {down }}$ be lifts of $S^{\text {up }}, S^{\text {down }}$ to $C$, respectively. See Figure 6. We will assume (only) that $F, F_{0}, F_{1}$ and each $S_{0}^{j}$ are least area surfaces in their isotopy classes.

Denote the components of $M(K) \backslash S_{0}^{j}$ by $M_{-}^{j}$ and $M_{+}^{j}$, with $M_{+}^{j}$ the component above $S_{0}^{j}$, i.e., the component containing $F_{1}$. (Note: we say that a surface in $C$ lies above $S_{0}^{j}$ if it lies in $M_{+}^{j}$ and below $S_{0}^{j}$ if it lies in $M_{-}^{j}$. It is easy to check, but important to realize, that if $S_{0}^{j}$ lies above $S_{0}^{k}$, then $S_{0}^{k}$ lies below $S_{0}^{j}$.) It follows from the construction of $S^{\text {up }}, S^{\text {down }}$ in Lemma 1 that $\tilde{S}^{\text {up }}, \tilde{S}^{\text {down }}$ are contained in $\tilde{T}^{\text {up }}$, $\tilde{T}^{\text {down }}$, respectively. Moreover, the push-off yielding $\tilde{T}^{u p}$ forces it to lie above $S_{0}^{i-1}$ and $S_{0}^{i+1}$, i.e., in the interior of $M_{+}^{i-1}$ and $M_{+}^{i+1}$. The push-off yielding $\tilde{T}^{\text {down }}$ forces it to lie below $S_{0}^{i-1}$ and $S_{0}^{i+1}$, i.e., in the interior of $M_{-}^{i-1}$ and $M_{-}^{i+1}$.

Here $S_{0}^{i}, S_{0}^{i+2}$ are disjoint from $S_{0}^{i-1} \cup S_{0}^{i+1}$ by Theorem 4 and hence, after isotopy if necessary, also from $\tilde{S}^{\text {up }} \cup \tilde{S}_{\widetilde{S}}^{\text {down }}$. We wish to show that $S_{0}^{i}, S_{0}^{i+2}$ either both lie below $\tilde{S}^{\text {down }}$ or both lie above $\tilde{S}^{\text {up }}$. Indeed, since $S_{0}^{i} \cap S_{0}^{i+2} \neq \emptyset$, we need only exclude 
the possibility that $S_{0}^{i} \cup S_{0}^{i+2}$ lie above $\tilde{S}^{\text {down }}$ but below $\tilde{S}^{\text {up }}$. Consider how $S_{0}^{i-1} \cup S_{0}^{i+1}$ lie with respect to $S_{0}^{i}$. Since $S_{0}^{i-1} \cap S_{0}^{i+1} \neq \emptyset$, they either both lie in $M_{-}^{i}$ or both lie in $M_{+}^{i}$. If

$$
S_{0}^{i-1} \cup S_{0}^{i+1} \subset M_{-}^{i}
$$

then $S_{0}^{i}$ lies above $S_{0}^{i-1} \cup S_{0}^{i+1}$ and hence above $\tilde{S}^{u p}$. If

$$
S_{0}^{i-1} \cup S_{0}^{i+1} \subset M_{+}^{i}
$$

then $S_{0}^{i}$ lies below $S_{0}^{i-1} \cup S_{0}^{i+1}$ and hence below $\tilde{S}^{\text {down }}$. A similar argument applies to $S_{0}^{i+2}$. Thus $S_{0}^{i}, S_{0}^{i+2}$ either both lie below $\tilde{S}^{\text {down }}$ or both lie above $\tilde{S}^{u p}$.

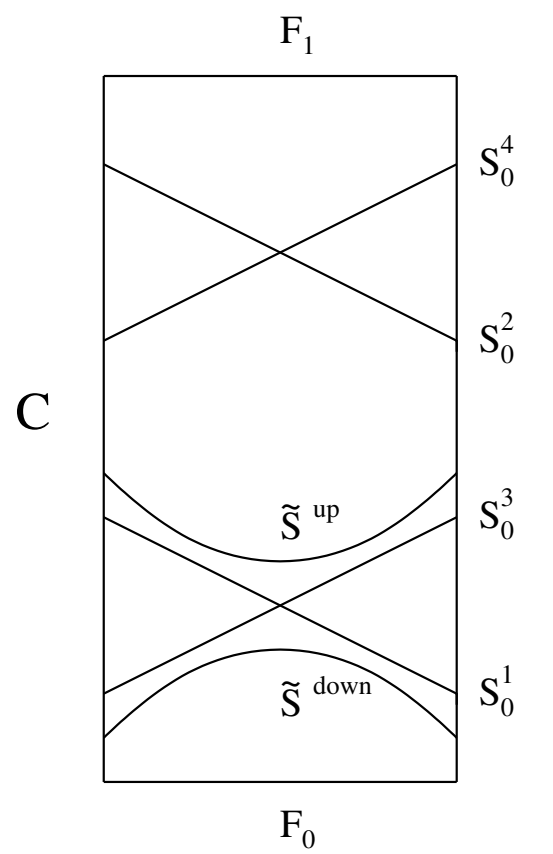

Figure 6: The product region

Since $v^{u p}=v^{\text {down }}=v, \tilde{S}^{u p}$ is isotopic to $F_{0}$ or $F_{1}$ as is $\tilde{S}^{\text {down }}$. It follows that either $S^{i-1}$ and $S^{i+1}$ lie in a product region and are hence isotopic, or that $S^{i}$ and $S^{i+2}$ lie in a product region and are hence isotopic. See Figure 6 . But this is impossible. Hence there are no homotopically nontrivial loops of length less than or equal to 4 in the link of $v$.

Lemma 4. A homotopically nontrivial loop in the link of a vertex in the Kakimizu complex must have length at least 6.

Proof: By Lemma 3, it suffices to show that there are no homotopically nontrivial loops of length 5. So suppose that $v^{1}, \ldots, v^{5}$ is a loop of length 5 in $X_{v}$. By the reasoning in Claim 1 in the proof of Theorem 6 , we may assume that $d_{K}\left(v^{i-1}, v^{i+1}\right)=2$ $\forall i$. (For otherwise the loop is homotopic, in $X_{v}$, to a loop of length 4 , and hence 
homotopically trivial by Lemma 3.) Endow $E(K)$ with a relative Riemannian metric and let $S^{1}, \ldots, S^{5}$ be relative least area representatives of $v^{1}, \ldots, v^{5}$. By Theorem 4 ,

$$
S^{i} \cap\left(S^{i+1} \cup S^{i-1}\right)=\emptyset \quad \forall i
$$

and

$$
S^{i-1} \cap S^{i+1} \neq \emptyset \quad \forall i .
$$

Recall the notation $M(K), F_{0}, F_{1}, C$ from the proof of Lemma 3 and let $S_{0}^{1}, \ldots, S_{0}^{5}$ be lifts of $S^{1}, \ldots, S^{5}$ to $C$. Then

$$
S_{0}^{i} \cap\left(S_{0}^{i+1} \cup S_{0}^{i-1}\right)=\emptyset \quad \forall i
$$

and

$$
S_{0}^{i} \cap S_{0}^{j} \neq \emptyset \text { for } j \neq i, i \pm 1 .
$$

Each $S_{0}^{i}$ is separating in $C$. In particular, $S_{0}^{2} \cap S_{0}^{5} \neq \emptyset$, so these two surfaces must either both lie above or both lie below $S_{0}^{1}$. Assume the former, as the other case will then follow by a symmetric argument. Also, $S_{0}^{3}$ must lie below $S_{0}^{2}$, in order to have nonempty intersection with $S_{0}^{1}$. Furthermore, $S_{0}^{4}$ must lie above $S_{0}^{3}$ in order to have nontrivial intersection with $S_{0}^{2}$, but below $S_{0}^{5}$, in order to have nontrivial intersection with $S_{0}^{1}$. See Figure 7 .

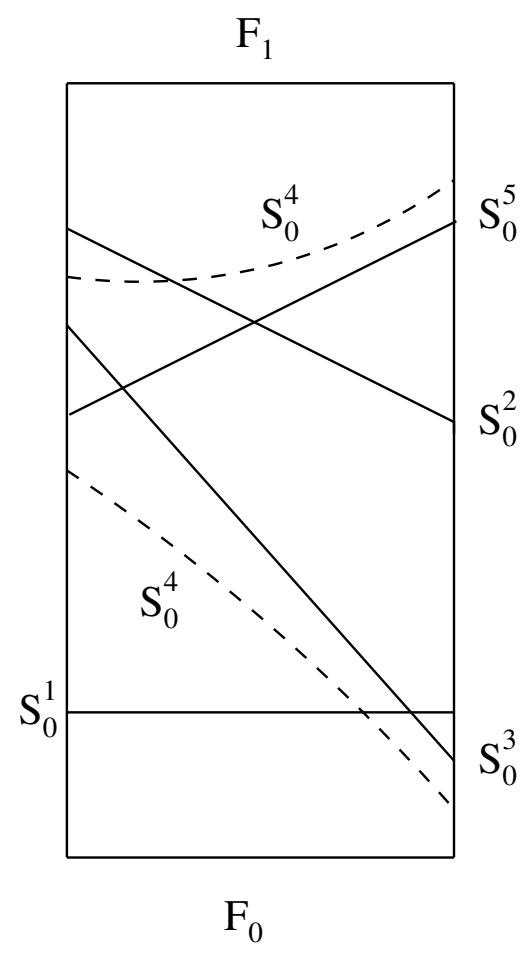

Figure 7: Five separating surfaces

Since $S_{0}^{3} \cap S_{0}^{5} \neq \emptyset$, this is impossible.

Theorem 8. If the Kakimizu complex of a knot $K$ is at most 2-dimensional, then it is contractible. 
Proof: Lemmas 3 and 4 establish the fact that links of vertices in the Kakimizu complex contain no nontrivial loops of lengths $\leq 5$. Thus the hypotheses of Theorem 7 are satisfied, whence the universal cover of the Kakimizu complex is contractible. Hence by Theorem 6 the Kakimizu complex is contractible.

\section{$7 \quad$ Further remarks}

To improve readability of this paper, the results have been stated and proved for knots in $\mathbb{S}^{3}$. However, they are equally valid for knots in homology 3-spheres. Furthermore, the Kakimizu complex is defined in terms of isotopy classes of minimal genus Seifert surfaces. A related complex is obtained by considering all isotopy classes of incompressible Seifert surfaces. The theorems and arguments used here are stated in terms of minimal genus Seifert surfaces. It seem likely that this assumption is unnecessary and that the analogous theorems hold for the more general complex as well.

The goal of proving contractibility of the Kakimizu complex may be out of reach, but there are natural questions to ask, now that simple connectedness is established. One such question is whether or not the Kakimizu complex is 2-connected. Indeed, this question is being pursued by Sakuma and Shackleton ([21]) who believe that it is 2-connected and that this can be established via the techniques used here.

Theorem 7 has a generalization, due to Januszkiewicz and Świạtkowski ([10]), to higher-dimensional simplicial complexes, although their notion of local $k$-largeness is more subtle. It is unclear if Lemmas 3 and 4 can be modified to fit their definition.

\section{Appendix: A compactness theorem for stable minimal surfaces by Michael Kapovich}

Let $M$ be a $\mathbb{P}^{2}$-irreducible compact Riemannian 3-manifold with smooth strictly convex boundary and $\mathcal{J}$ a compact family of smooth curves on $\partial M$. In the setting here we are mostly interested in the case where $\partial M$ is a single torus and $\mathcal{J}$ is a smooth foliation of $\partial M$ by closed curves. Let $S$ be a compact connected surface, possibly with boundary.

Given a smooth proper embedding $f:(S, \partial S) \rightarrow(M, \partial M)$ we let $[f]$ denote its (proper) isotopy class (here we are not fixing the boundary value $f \mid \partial S$ ). From now on, we will assume that the isotopy classes $[f]$ are such that for each boundary component $\partial_{i} M$ of $M, f^{-1}\left(\partial_{i} M\right)$ is a single component of $\partial S$ and that the surface $f(S)$ is incompressible. It follows that for each $f$ as above, there exists a least area (parameterized) surface in the set

$$
\{g \in[f]: g|\partial S=f| \partial S\},
$$

see $[8$, Theorem 6.12$]$. Such a surface is necessarily a stable minimal surface. 
Define the "moduli space" $\mathcal{M}([f])$ of stable minimal surfaces in the given proper isotopy class $[f]$ subject to the condition that $f \mid \partial S$ is a parameterized multi-curve in $\mathcal{J}$. Here we identify parameterized surfaces which differ by a reparameterization of $S$. We let $\mathcal{M}=\mathcal{M}(S)=\cup_{[f]} \mathcal{M}([f])$ be the space of all stable embedded minimal surfaces of the given topological type. We give $\mathcal{M}$ the $C^{1}$-topology. Given a number $a$ we let $\mathcal{M}_{a}$ denote the subset of $\mathcal{M}$ consisting of surfaces of area $\leq a$.

Proposition 9. If $S$ has nonempty boundary, then the space $\mathcal{M}_{a}$ is compact.

When $\partial S$ is empty, a similar compactness result holds by a theorem of Nakauchi. In [13], Nakauchi uses Schoen's estimates [20] on the norm of the 2-nd fundamental form of stable minimal surfaces away from the boundary of $M$ to conclude that sequences of stable minimal surfaces admit convergent subsequences, except that the limiting surfaces in [13] may fail to be embedded but appear as 2-fold coverings of embedded surfaces.

Proof: In the case of surfaces with boundary we modify Nakauchi's argument as follows: Given a sequence of minimal surfaces $f_{i}: S \rightarrow M$ whose boundary values are in $\mathcal{J}$ and whose area is $\leq a$, a theorem of Anderson [3, Theorem 3.1] tells us that there exists a subsequence $f_{i_{j}}$ so that the sequence $f_{i_{j}}(S)$ converges to a minimal surface $\Sigma \subset M$ whose boundary is in $\mathcal{J}$. The surface $\Sigma$ need not be of the same topological type as $S$. However, the convergence of the surfaces is smooth away from a finite subset $x_{1}, \ldots, x_{m} \in \Sigma$. Moreover, Anderson proves [3, Paragraph 4 of the proof of Theorem 3.1] that all the points $x_{i}$ belong to the interior of $M$. Thus we can apply Schoen's estimates [20] to each point $x_{i} \in \operatorname{int}(M)$ in the same manner as Nakauchi does, provided that the $f_{i}(S)$ are stable minimal surfaces. Schoen's estimates imply smooth convergence at the points $x_{1}, \ldots, x_{m}$. Therefore, the maps $f_{i_{j}}: S \rightarrow M$ converge smoothly to a covering map $f: S \rightarrow \Sigma$. Suppose that $f$ is a nontrivial covering, then its restriction to $\partial S$ is also nontrivial. However, compactness of $\mathcal{J}$ implies that the maps $f_{i_{j}}: \partial S \rightarrow \partial M$ converge to an embedding. This is a contradiction. Therefore, $f$ is $1-1$.

Remark 5. If $M$ is a closed 3-manifold with a bumpy Riemannian metric, Colding and Minicozzi proved in [5] that the space of (not necessarily stable) minimal surfaces of uniformly bounded area is finite: Bumpiness of the metric is used to ensure that the limiting minimal surface $\Sigma$ has no nontrivial Jacobi fields. The same argument can be used in conjunction with the above proposition to ensure finiteness of $\mathcal{M}_{a}$, provided that the metric on $M$ is chosen to be bumpy on the interior of the manifold.

Corollary 10. $\mathcal{M}_{a}$ splits as a disjoint union of finitely many open and closed subsets $\mathcal{M}_{a}([g])$ each of which consists of isotopic surfaces.

Proof: Let $g$ be a stable minimal surface in $\mathcal{M}_{a}$ and let $f_{k}: S \rightarrow M$ be a sequence of surfaces in $\mathcal{M}_{a} \backslash \mathcal{M}_{a}([g])$. Suppose that the sequence $f_{k}: S \rightarrow M$ converges to $f: S \rightarrow M$. Then $f$ is isotopic to each $f_{k}$ for $k$ sufficiently large. In particular, $f$ cannot be in $\mathcal{M}_{a}([g])$. It follows that the subsets $\mathcal{M}_{a}([g])$ are open. Finiteness of the number of these sets follows from the compactness of $\mathcal{M}_{a}$. 
Corollary 11. Each $\mathcal{M}([f])$ contains an area-minimizer. In particular, each class $\{g \in[f]: g \mid \partial S \in \mathcal{J}\}$ admits an area-minimizer.

Proof: Area is a continuous function, therefore the corollary follows from the compactness of $\mathcal{M}_{a}([f])$.

We now assume that $\partial M$ is a union of tori. We say that a Riemannian metric on $M$ is relative if $M$ has a strictly convex boundary. It is easy to see that $M$ always admits a relative Riemannian metric: Start with a metric on $T^{2} \times[0,1]$, where $T^{2} \times\{0\}$ strictly convex. Identify $T^{2} \times[0,1]$ with neighborhoods of boundary tori $\partial_{i} M$ of $M$, where $\partial_{i} M$ is identified with $T^{2} \times\{0\}$. Then extend the metric on a neighborhood of $\partial M$ arbitrarily to the rest of $M$. Fix a $C^{1}$-foliation $\mathcal{J}$ of $\partial M$ by closed curves.

The area minimizers in the isotopy classes $\mathcal{M}([f])$ will be called relative least area surfaces (the word "relative" refers to the fact that we are assuming that $f(\partial S) \in \mathcal{J}$ ). We now compare our setup with that of [7].

In our boundary conditions for relative least area surfaces we are using boundary curves in $\mathcal{J}$ rather than using a free boundary condition as is done in [7]. However, since curves in $\mathcal{J}$ are either disjoint or equal, this ensures that the cut-and-paste arguments used in [7] preserve our boundary conditions. With this in mind, the arguments in [7] go through with our set-up. In particular, we obtain:

Theorem 12. Let $f_{i}: S_{i} \rightarrow M, i=1, \ldots, n$, be incompressible surfaces which are pairwise non-isotopic and pairwise disjoint. Let $g_{i}: S_{i} \rightarrow M, i=1, \ldots, n$ be relative area minimizers in the isotopy classes of $f_{i}, i=1, \ldots, n$. Then $g_{1}\left(S_{1}\right), \ldots, g_{n}\left(S_{n}\right)$ are also pairwise disjoint.

We now assume that $M$ is diffeomorphic to the exterior of a knot in $\mathbb{S}^{3}, \mathcal{J}$ is a foliation of $\partial M$ by preferred longitudes and the surface $S$ has a single boundary component.

Fix a number $n$ and let $\mathcal{L} \subset \mathcal{M}^{n}$ denote the subset of $n$-tuples represented by parameterized minimal genus Seifert surfaces $\left(f_{1}, \ldots, f_{n}\right)$, so that:

a) $d_{K}\left(\left[f_{i}(S)\right],\left[f_{i+1}(S)\right]\right)=1, i$ is taken $\bmod n$. Here $d_{K}$ is the Kakimizu distance and the isotopy class $\left[f_{k}(S)\right]=v_{k}$ represents a vertex in the Kakimizu complex.

b) The loop in the 1-skeleton of the Kazimizu complex represented by the vertices $v_{1}, \ldots, v_{n}$ is homotopically nontrivial in the Kazimizu complex.

It follows immediately from Corollary 10 that $\mathcal{L}$ is closed in $\mathcal{M}^{n}$. Define the area functional $A: \mathcal{M}^{n} \rightarrow \mathbb{R}_{+}$,

$$
A\left(f_{1}, \ldots, f_{n}\right)=\sum_{i=1}^{n} A\left(f_{i}(S)\right) .
$$

We then obtain the following:

Corollary 13. The functional $A \mid \mathcal{L}$ attains a minimum.

Proof: It is clear that $A$ is continuous and positive. By Proposition 9, $A$ is proper. Since $\mathcal{L}$ is closed in $\mathcal{M}^{n}$, the restriction $A \mid \mathcal{L}$ is proper as well. Therefore, $A \mid \mathcal{L}$ attains its minimum. 


\section{References}

[1] C. Adams: The knot book. An elementary introduction to the theory of knots. American Mathematical Society, Providence, RI, 2004.

[2] W. R. Alford: Complements of minimal spanning surfaces are not unique, Ann. Math. 91 (1970) 419-424.

[3] M. Anderson: Curvature estimates for minimal surfaces in 3-manifolds, Ann. Sci. École Norm. Sup. (4), 18 (1985), pp. 89-105.

[4] M. Bridson, A. Haefliger: Metric spaces of non-positive curvature. Grundlehren der Mathematischen Wissenschaften [Fundamental Principles of Mathematical Sciences], 319. Springer-Verlag, Berlin, 1999.

[5] T. Colding, W. Minicozzi, II: Embedded minimal surfaces without area bounds in 3-manifolds, in Geometry and topology: Aarhus (1998), vol. 258 of Contemp. Math., Amer. Math. Soc., Providence, RI, 2000, pp. 107-120.

[6] J. R. Eisner: Knots with infinitely many minimal spanning surfaces, Trans. Amer. Math. Soc. 229 (1977) 329-349.

[7] M. Freedman, J. Hass, G. P. Scott: Area minimizing incompressible surfaces, Invent. Math. 71 (1983) 609-642.

[8] J. Hass, P. Scott: The existence of least area surfaces in 3-manifolds, Trans. Amer. Math. Soc., 310 (1988), pp. 87-114.

[9] M. Hirasawa, M. Sakuma: Minimal genus Seifert surfaces for alternating links, Proceedings of Knots 96 (1997) 383-394.

[10] T. Januszkiewicz, J. Świątkowski: Simplicial nonpositive curvature, Publications Mathématiques de l'Institut des Hautes Études Scientifiques, Volume 104, Number 1, November 2006, pp. 1-85.

[11] O. Kakimizu: Finding disjoint incompressible spanning surfaces for a link, Hiroshima Math. J. 22 (1992) 225-236.

[12] W.B.R. Lickorish: An introduction to knot theory. Graduate Texts in Mathematics 175. Springer-Verlag, New York, 1997.

[13] N. Nakauchi: Compactness of incompressible stable minimal surfaces without boundary, J. of Mathematics of Kyoto University, 30 (1990), pp. 343-346.

[14] U. Oertel: On the existence of infinitely many essential surfaces of bounded genus, Pacific J. Math. 202, no. 2 (2002) 449-458.

[15] R. Pelayo: Diameter bounds on the complex of minimal genus Seifert surfaces for hyperbolic knots, thesis at California Institute of Technology, available at http://thesis.library.caltech.edu/2446/. 
[16] D. Rolfsen: Knots and links. Mathematics Lecture Series 7. Publish or Perish, Inc., Huston, TX, 1990.

[17] M. Sakuma: Minimal genus Seifert surfaces for special arborescent links, Osaka J. Math. 31 (1994) 861-905.

[18] M. Sakuma, K. Shackleton: On the distance between two Seifert surfaces of a knot, Osaka J. Math. 46 (2009) 203-221.

[19] M. Scharlemann, A. Thompson: Finding disjoint Seifert surfaces, Bull. London Math. Soc. 20 (1988) 61-64.

[20] R. Schoen: Estimates for stable minimal surfaces in three dimensional manifolds, in Seminar on minimal submanifolds, vol. 103 of Annals of Mathematics Studies, Princeton University Press, 1983, pp. 111-126.

[21] K. Shackleton: private communication.

[22] Y. Tsutsumi: Hyperbolic knots with a large number of disjoint minimal genus Seifert surfaces, Tokyo J. Math. 31 (2008), no. 1, 253-258.

[23] R. Wilson: Knots with infinitely many incompressible Seifert surfaces, J. Knot Theory Ramifications 17 (2008), no. 5, 537-551.

Department of Mathematics

1 Shields Avenue

University of California, Davis

Davis, CA 95616

USA 